\title{
A Re-Analysis of the X-ray Spectrum of HR 1099 and Its Implications for Coronal Abundances
}

\author{
STEPHEN A. DRAKE, ${ }^{1,2}$ KULINDER P. SINGH, ${ }^{1,3}$ \\ AND NICHOLAS E. WHITE ${ }^{1}$ \\ ${ }^{1}$ HEASARC, Code 668, NASA/GSFC, Greenbelt, MD 20771, USA \\ ${ }^{2}$ USRA, Code 610.3, NASA/GSFC, Greenbelt, MD 20771, USA \\ ${ }^{3}$ TIFR, Homi Bhabha Road, Colaba, Bombay, 400 005, India
}

\begin{abstract}
We have analyzed archival X-ray spectra of the RS CVn binary system HR 1099 in an attempt to see if we can obtain a consistent picture of the state of the X-ray emitting plasma. We have modeled six spectra obtained by the Exosat ME and LE telescopes, two spectra obtained by the Einstein SSS and MPC instruments, as well as a more recent ROSAT PSPC spectrum. We find that these spectra are in general poorly fitted by solar-abundance Raymond and Smith or Mewe and Kaastra thermal plasma models, and that no simple combination of these models significantly improves these fits: the observed continuum is too strong relative to the line features. We find acceptable fits for thermal models with two or more components in which the heavy elements are depleted by a factor of 2 to 4 relative to their solar photospheric values. These results are consistent with those obtained from the analysis of higher-resolution $E U V E$ and $A S C A$ spectra of active binary and single stars. We discuss the implications of these findings on ongoing analyses of the EUV spectra of HR 1099 and other RS CVn binaries.
\end{abstract}

\section{Introduction}

Recent results from moderate-resolution X-ray spectroscopy with $A S C A$ and highresolution EUV spectroscopy with $E U V E$ of the coronae of active, late-type stars have yielded surprising results concerning the elemental abundances of these high-temperature plasmas. Most of the stars for which detailed analyses have been obtained appear to show a general underabundance of metals, relative to their solar photospheric values of factors from $\sim 2-6$ (e.g., White et al. 1994; Drake et al. 1994; Singh et al. 1995a; Stern et al. 1995; Rucinski et al. 1995). This result is quite unexpected, since most of these solar neighborhood stars are thought to have photospheric abundances similar to the sun. The solar corona does indeed exhibit a different pattern of abundances than the solar photosphere, but in a completely opposite sense to that inferred for stellar coronae: elements like Fe with first-ionization potentials (FIPs) less than $\sim 10 \mathrm{eV}$ are enhanced by factors from $\sim 1-10$ compared to their photospheric values (e.g., Anders \& Grevesse 1989; Feldman 1992).

Before attempting to explain the inferred peculiar abundances found in stellar coronae, it is essential that their reality be firmly established. In particular, why was evidence for this metal-deficiency effect not inferred from previous (admittedly generally either lower spectral-resolution and/or signal-to-noise) X-ray observations of stellar coronae? To answer this question, we examine Einstein, EXOSAT, and ROSAT spectra of the prototypical RS CVn binary star HR 1099 (V711 Tau). As secondary goals of the present study we want to (i) refute or verify the existence of a high-temperature $\left(T_{e} \gg 10^{7.5} \mathrm{~K}\right)$ plasma component of its corona, such as has been inferred might be present from analyses of $E U V E$ spectra of similar active stars, by searching for a high-energy tail in the 3 to $20 \mathrm{keV}$ region of its $\mathrm{X}$-ray spectrum; and (ii) derive best-fit models from earlier epoch 
observations of HR 1099 that can be compared with more recent $E U V E$ and $A S C A$ observations of this system.

\section{Observations and Analysis}

The following archival X-ray spectra of HR 1099 have been analyzed:

(i) Two observations by the Einstein Observatory Solid State Spectrometer (SSS) (spectral resolution $\Delta E$ of $\sim 160 \mathrm{eV}$ covering 0.5 to $4.5 \mathrm{keV}$ ) and the Monitor Proportional Counter (MPC) (8 logarithmically spaced energy channels covering the 1 to 20 $\mathrm{keV}$ band) made in 1979, with a total exposure of $\sim 10$ kiloseconds $(\mathrm{ks})$.

(ii) Six observations made by the EXOSAT Observatory Low Energy (LE) telescope and Medium Energy (ME) proportional counter array in the period 1983 to 1986, with a total exposure of $\sim 200 \mathrm{ks}$. The LE essentially measures the X-ray flux in the 0.1 to $2.0 \mathrm{keV}$ band, while the ME covers the 1 to $20 \mathrm{keV}$ band with an energy resolution increasing from $0.5 \mathrm{keV}$ at low energies to $2.0 \mathrm{keV}$ at high energies.

(iii) An observation made by the ROSAT Observatory Position Sensitive Proportional Counter (PSPC) in 1992 with an exposure time of $2.7 \mathrm{ks}$.

We have used the XSPEC (Version 8.50) spectral analysis package to fit the data with the Mewe-Kaastra, or "meka" spectral models (Mewe et al. 1985; Kaastra 1992) for thermal-equilibrium plasmas. (Similar results are obtained using the Raymond \& Smith model (Raymond 1990; Raymond \& Smith 1977), except for a small difference in the derived temperatures). We used the "meka" model for ease of comparison with our earlier work on active, stars where we also found somewhat better fits using "meka" model (Drake et al. 1994).

We tried (a) single-temperature or isothermal plasma models, (b) a model consisting of two or more discrete plasma components at different temperatures, and (c) a plasma model with continuous emission measure (CEM) distribution which is a power-law function of the temperature of the type, $\operatorname{EM}(T) \propto\left(T / T_{\max }\right)^{\alpha}$, where $T_{\max }$ is the maximum temperature of the plasma and $\alpha$ is the slope of the emission measure (EM) distribution (Schmitt et al. 1990). The last of these is a good approximation to models that have been used to describe the EM distribution of individual coronal loops on the sun (e.g., Antiochos \& Noci 1986). It has also been used to fit the X-ray emission from stellar coronae which would normally involve an ensemble of such loops (e.g., Stern et al. 1986; Schmitt et al. 1990). We have kept $\alpha$ and $\mathrm{T}_{\max }$ as free parameters. The elemental abundances in the plasma for all of these different models, were varied with respect to the solar photospheric values taken from Anders \& Grevesse (1989).

\section{Results}

A subset of the results of this analysis are given in Table 1, where we list the parameters of the best-fit 2-component models and their corresponding reduced chi-squared fit statistics, $S$. Some examples of the best-fit models together with the observed spectra are shown in figures 1 and 2.

The EXOSAT LE + ME, Einstein SSS + MPC, and ROSAT PSPC spectra are all best fitted by two-component plasma emission models with temperatures of $0.6-0.7 \mathrm{keV}$ and 2-3 keV, and having sub-solar abundances. There is no evidence for significant amounts of hotter $\left(T_{e} \sim 10^{8} \mathrm{~K}\right)$ plasma being present. We find that discrete (two) component plasma emission models can usually better fit the data than the power-law CEM models based on models of single solar loops; the latter models are even found to be unacceptable in several cases. Given the low-resolution nature of the spectra discussed here, we have 


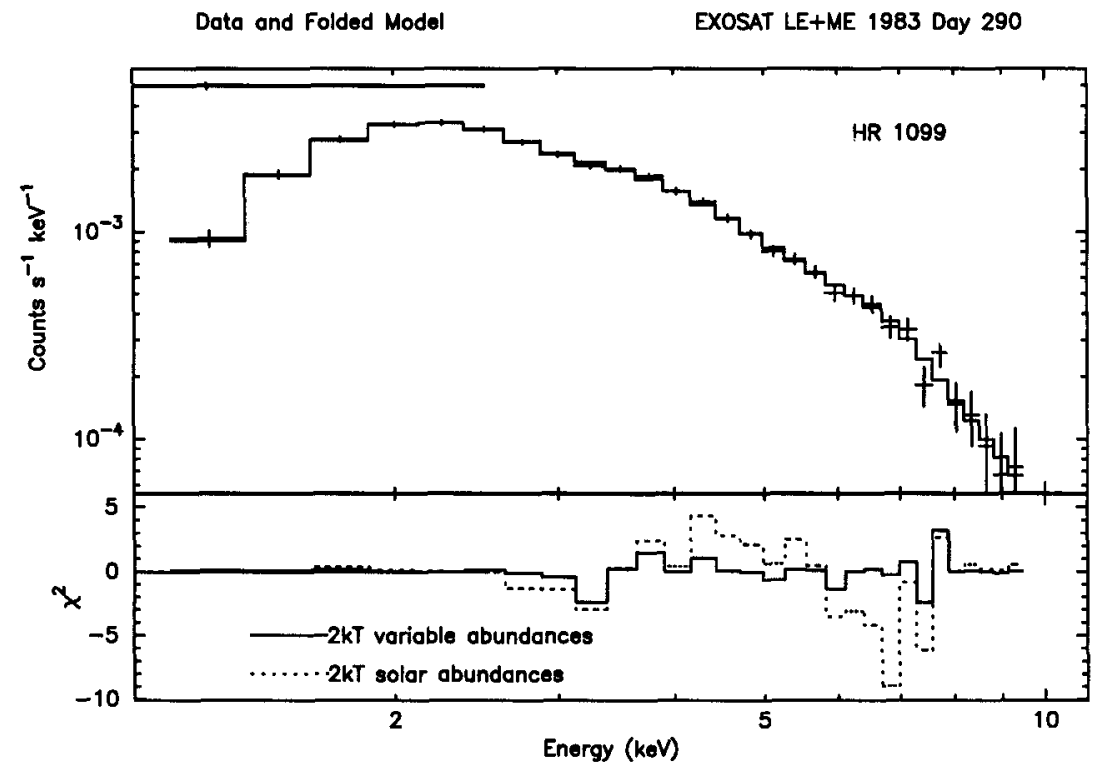

FIGURE 1. The observed spectrum of HR 1099 obtained with the EXOSAT LE and ME (the crosses) on 1983 , day 290 , compared to the best-fit $2-T$ variable abundance model (the histogram). The lower panel shows the $\chi^{2}$ residuals both for this model and, for comparison, the best-fit $2-T$ solar abundance model.

TABLE 1. Summary of Results of Two-Component Models

\begin{tabular}{ccllllllr} 
& \multicolumn{4}{c}{ Best solar abund. model } & \multicolumn{3}{c}{ Best variable abund. model } \\
Data Set & Date & $T$ (low) & $T$ (high) & S & $T$ (low) & $T$ (high) & $f$ & S \\
SSS + MPC & 1979.222 & 0.66 & 3.6 & 1.42 & 0.68 & 3.4 & 0.54 & 1.31 \\
SSS + MPC & 1979.223 & 0.67 & 4.0 & 1.37 & 0.68 & 3.9 & 0.49 & 1.23 \\
LE + ME & 1983.290 & 0.41 & 2.7 & 1.97 & 1.34 & 4.2 & 0.24 & 0.60 \\
LE + ME & 1985.267 & 0.92 & 2.3 & 1.45 & 1.26 & 5.0 & $<0.88$ & 1.35 \\
LE + ME & 1985.267 & 1.06 & 2.5 & 0.94 & 1.25 & 3.3 & $<0.87$ & 0.85 \\
LE + ME & 1986.033 & 0.54 & 2.2 & 1.97 & 0.73 & 2.3 & $<0.67$ & 1.70 \\
LE + ME & 1986.034 & 0.45 & 1.9 & 2.60 & 1.17 & 2.7 & $<0.19$ & 1.02 \\
LE + ME & 1986.035 & 0.57 & 2.0 & 1.43 & 0.96 & 2.2 & $<0.63$ & 1.21 \\
PSPC & 1992.026 & 0.19 & 1.0 & 6.70 & 0.57 & 1.3 & 0.29 & 1.21 \\
\hline
\end{tabular}

limited our consideration to models with either solar (photospheric) abundances or those having all the non-hydrogenic atomic abundances varying from solar by a common factor $f$. Not surprisingly, the introduction of the latter additional free parameter does improve the fits to the observed spectra: some spectra, however, can simply not be fit by solarabundance plasmas with the admittedly simple thermal structures that we have tested. The abundance results can be summarized thus: (i) In all cases the inferred values of the abundance factor $f$ are subsolar, with best-fit values in the range of $0.2-\leq 0.9$; (ii) The abundance factor $f$ appears to be variable: some spectra appear to be only mildly metal-deficient, while others (e.g., the 1986 Day 33/34 EXOSAT spectrum) appear to be 


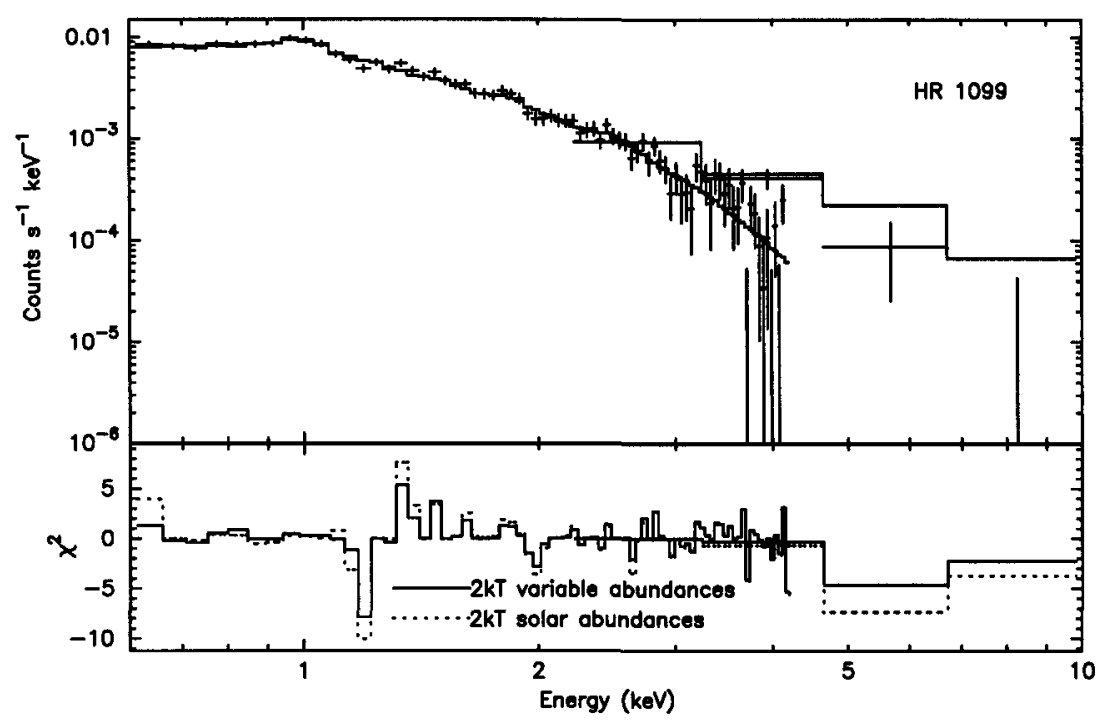

FIGURE 2. Similar to Fig. 1, but showing the observed and model spectra for the Einstein SSS and MPC data obtained on 1979, day 223.

much more depleted; and (iii) The abundance results are broadly compatible with those that we and others have inferred from $A S C A$ spectra of active, late-type stars.

\section{Discussion}

Thus, the analysis of the archival X-ray spectra of HR 1099 has confirmed the metaldeficiency effect seen in $A S C A$ and $E U V E$ spectra of active stars. The reason that these abundance peculiarities were not inferred 15 years ago is apparently that most previous studies of coronal spectra have been made without allowing the abundances to vary from their solar photospheric values. The next question to address is whether these non-solar abundances are intrinsic (i.e., real) or are an artifact somehow resulting from our analysis. We believe that we have demonstrated here that they are not due to an instrumental artifact, since similar results are obtained from several different X-ray instruments of widely differing properties such as spectral resolution.

If there is a problem then, it must be associated with the coronal plasma models used in the fits. Indeed, it is known that the 'meka' and Raymond and Smith codes incorporated into the present version of XSPEC poorly represent the the Fe $\mathrm{L}$ shell region of 0.7 to $1.4 \mathrm{keV}$, due to a large number of errors and omissions, and that strong lines in this complex can be over- or underestimated by as much as factors of 2 or 3 . Until we have the next-generation 'meka' code available for comparison, it is impossible for us to say how the problems with the current-generation codes will affect the inferred Fe abundance. For example, in the worst-case scenario, if the emission from the whole Fe L complex has been overestimated by a factor of 3 , then the underabundance of $F e$ that has been derived herein and in previous papers discussing $A S C A$ spectra of late-type stars would 
be spurious. In fact, according to Liedahl et al. (1995), the picture is not as simple as that: some lines are underestimated, while some lines are over-estimated, so that, based on this preliminary report, it seems difficult to attribute the factor of 3 deficiency in $\mathrm{Fe}$ (relative to solar photospheric) found here to this known problem. Furthermore, similar Fe underabundances are inferred from studies of the $\mathrm{Fe} \mathrm{K} 6.7 \mathrm{keV}$ feature for which the atomic physics is known to be fairly accurate.

The other major result of this study is that there is no upturn in the emission measure distribution at temperatures $T_{e} \geq 10^{8} \mathrm{~K}$. X-ray spectra would easily detect this material if it were present: it is not. As is discussed in more detail in Singh et al. (1995b), there is a relatively small amount of information that can be derived about the detailed temperature structure of coronae from low or even moderate spectral resolution X-ray spectra: $E U V E$ spectra, if of high enough signal-to-noise, are clearly superior in this regard. The best fits to X-ray spectra of cool stars are simple 2-temperature models, although poorer, but still acceptable fits can usually be obtained by more complex continuous emission measure models. However, these same analyses also show that the elemental abundances inferred from X-ray spectra are in general very insensitive to the assumed temperature structure. Thus, it is much easier to derive information on abundances from $A S C A$ spectra than $E U V E$ spectra, both because of this reason, as well as the presence of strong features due to $\mathrm{Si}, \mathrm{Mg}, \mathrm{S}, \mathrm{Ar}$, and $\mathrm{Ca}$ in the $A S C A$ spectral range.

This research has made use of data obtained through the High Energy Astrophysics Science Archive Research Center Online Service, provided by the NASA-Goddard Space Flight Center.

\section{REFERENCES}

Anders, E. \& Grevesse, N. 1989, Geochimica et Cosmochimica Acta, 53, 197

AnTiochos, S. K. \& NocI, G. 1986, ApJ, 301, 440

DraKe, S. A. ET AL. 1994, ApJ, 436, L87-L90

Feldman, U. 1992, Physica Scripta, 46, 202

KAASTRA, J. 1992, An X-ray Spectral Code for Optically thin plasmas, SRON-Leiden

LIEDAHL, D. A. ET AL. 1995, ApJ, 438, L115-L118

MEWE, R. ET AL. 1985, A\&ApS, 62, 197

RAYMOND, J. C. 1990, private communication

RAYMond, J. C. \& SMith, B. W. 1977, ApJS, 35, 419

RUCINSKI, S. M. ET AL. 1995, this volume

SchMitT, J. H. M. M. ET AL. 1990, ApJ, 365, 704

SINGH, K. P. ET AL. 1995a, ApJ, in press

SiNGH, K. P. ET AL. 1995b, ApJ, submitted

STERN, R. A. ET AL. 1986, ApJ, 305, 417

STERN, R. A. ET AL. 1995, ApJ, 444, L45-L48

WhITE, N. E. ET AL. 1994, PASJ, 46, L97-L100 